\title{
Effect of Thermal Discharges on the Fish Assemblages of a Nuclear Power Plant in Northern Taiwan
}

\author{
Ching-Yi Chen \\ Reseach Center for Biodiversity, Academia Sinica, Taipei, Taiwan 115.
}

Kwang-Tsao Shao

Reseach Center for Biodiversity, Academia Sinica, Taipei, Taiwan 115., zoskt@gate.sinica.edu.tw

Yueh-Yuan Tu

Department of Industrial Safety and Environmental Protection, Taiwan Power Company, Taipei, Taiwan 100.

Follow this and additional works at: https://jmstt.ntou.edu.tw/journal

Part of the Terrestrial and Aquatic Ecology Commons

\section{Recommended Citation}

Chen, Ching-Yi; Shao, Kwang-Tsao; and Tu, Yueh-Yuan (2004) "Effect of Thermal Discharges on the Fish Assemblages of a Nuclear Power Plant in Northern Taiwan," Journal of Marine Science and Technology: Vol. 12: Iss. 5, Article 6. DOI: $10.51400 / 2709-6998.2261$

Available at: https://jmstt.ntou.edu.tw/journal/vol12/iss5/6

This Research Article is brought to you for free and open access by Journal of Marine Science and Technology. It has been accepted for inclusion in Journal of Marine Science and Technology by an authorized editor of Journal of Marine Science and Technology. 


\title{
EFFECT OF THERMAL DISCHARGES ON THE FISH ASSEMBLAGES OF A NUCLEAR POWER PLANT IN NORTHERN TAIWAN
}

\author{
Ching-Yi Chen*, Kwang-Tsao Shao*, and Yueh-Yuan Tu**
}

Key words: thermal plume, thermal pollution, coral fish, ecological monitoring.

\section{ABSTRACT}

The purpose of this paper is to study whether the thermal plume can affect the fish assemblages in the waters around the outlet area of the Second Nuclear Power Plant located at Kuosheng, a coastline between Yehliu and Chinshan village, northern coast of Taiwan. Both experimental and control stations of underwater census for reef fishes and of drift net sampling for pelagic or demersal fishes above sandy bottom were monitored four times per year from March 2001 to September 2004. The results show that no significant dif-ferences were found between the fish assemblages of the thermal waters and normal ambient waters for both coral reef fishes and pelagic or demersal fishes. It was probably due to microhabitat difference rather than water difference because the above fishes are mostly living near bottom where the water temperature are similar to each others for these two stations. On the contrary, the seasonal effect due to low water temperature in the winter had greater influence on the fish assemblages than the effect of thermal plume.

\section{INTRODUCTION}

Three Nuclear Power Plants (NPP) have been operating since 1977,1981 and 1984 respectively in northern $\left(1^{\text {st }}\right.$ and $2^{\text {nd }}$ NPP) and southern $\left(3^{\text {rd }}\right.$ NPP) Taiwan. The fourth Nuclear Power Plant is still constructing at Yenliao, northeastern coast of Taiwan. It is always in debate whether the thermal plume affects the fish resources in the waters around NPP. The water temperature difference $(\Delta \mathrm{T})$ between inlet and outlet area in Taiwan was designed up to $7-12^{\circ} \mathrm{C}$, and the $2^{\text {rd }}$ NPP was the highest, i.e., $10-12^{\circ} \mathrm{C}$. Thermal waters could affect metabolism, growth, feeding, spawning and behavior of marine organisms as well as to their

Paper Submitted 11/16/04, Accepted 02/14/05. Author for Correspondence: Kwang-Tsao Shao.E-mail: zoskt@gate.sinica.edu.tw.

*Reseach Center for Biodiversity, Academia Sinica, Taipei, Taiwan 115.

**Department of Industrial Safety and Environmental Protection, Taiwan Power Company, Taipei, Taiwan 100. community structure and ecosystem $[1,3,4]$. In India, massive death of crabs and many macrobenthos were observed in the hot season [12, 13]. In France, seaweed assemblages encrusted on rocky shore changed when water temperature increased slightly $0.5-1.0^{\circ} \mathrm{C}$ [15]. In winter, thermal discharges of power plants affected the assemblage structure, recruitment, mortality, demography, spawning age, gonad development, and net production of fishes in Baltic Sea [10]. Cool shock due to shut down of power plant in winter also gave great impact to some fish species [14]. On the contrary, the assessment report from several large power plants along the coast in Italy did not detect the thermal effect on the community structures of phytoplankton, benthic organisms and fishes [1]. Nevertheless, it was pointed out that their study sites were too far away from the outlet area.

In Taiwan, it is well known that thermal plume of the 3rd NPP let the corals bleaching in the shallow waters near the outlet area $[3,11]$. The thornfishes (Therapon jurba) and the large scale mullet (Liza macrolepis) were malformed inside the outlet bay of the $2^{\text {nd }}$ NPP every summer since 1993 [7, 9]. It was because of Vitamin C deficiency which caused fish backbone to curve [5]. However, it still has no concrete evidence which proves that the thermal discharges will or will not affect the fish assemblages in Taiwan except typhoon effects at the $3^{\text {rd }}$ NPP [7]. Although no thermal impacts were found at both the $1^{\text {st }}$ and $2^{\text {nd }}$ NPP in earlier ecological assessment before 1990. It has been pointed out that both experimental (at Yehliu) and control stations (at Kueho) chosen during that time were too far away from the outlet areas of the $1^{\text {st }}$ and the $2^{\text {nd }}$ NPP. [6]. The area which could be affected by thermal plume should be inside the range of 500-1,000 meters away from the outlet. Therefore, the current study moved both experimental and control stations back to the areas very close to the outlet area of the $2^{\text {nd }}$ NPP. Both coral reef fish and pelagic or demersal fish assemblages were monitored and compared in order to find out the effect of thermal discharge to the local fish assemblages. 


\section{MATERIALS AND METHODS}

\section{Study area and sampling design}

According to the regulation for controlling thermal discharges by EPA, Taiwan, the rise of water temperature on the borderline of $500 \mathrm{~m}$ radius off outlet can not exceed $4^{\circ} \mathrm{C}$ than ambient water temperature. The hydrological simulation of thermal plume at the $2^{\text {nd }}$ NPP after reconstruction of the outlet structure in 1993, the water temperature surrounded indeed decreased and met the requirement of EPA. In other words, the area about 500-1,000 m away from the outlet bay should have had no impact from warmer waters, especially in the waters below 2-3 meters because heat waters only float in the upper layer $[6,11]$. Thus, several new monitoring stations located near outlet bay were chosen to replace those old stations since 2001.

For monitoring coral reef fishes, species and abundance data were recorded by two divers using a $100 \mathrm{~m}$ transect line ( $3 \mathrm{~m}$ each side) along the jetty of outlet bay as the experimental station ( $25^{\circ} 12^{\prime} 17^{\prime \prime}, \mathrm{E} 121^{\circ} 39^{\prime}$ $\left.50^{\prime \prime}\right)$, and another jetty about $1,300 \mathrm{~m}$ away as the control station (Fig. 1). The survey was carried four times per year during March 2001 and September 2004. The underwater topography, substratum, and water depth around 6-7 meters were very similar to each others between these two transects.

For monitoring pelagic or demersal fishes in sandy/ muddy bottom, "three-layer" drift nets operated by fishermen were deployed parallel in both $300 \mathrm{~m}$ and 800 $\mathrm{m}$ away from outlet bay as the experimental and controlled station respectively (Fig. 1). The sampling frequency of drift nets was the same as underwater census. All specimens collected by drift nets were brought back to laboratory for further species identification, counting individual numbers and body weight measurement.

\section{Data analysis}

The Paired t test was used to analyze whether the species and individual number between experimental and control stations were significantly different seasonally. Multivariate statistical analysis was applied to analyze the community structure difference by using PRIMER V5.2 [2]. The data of fish species and individual numbers were transformed by $\log (1+x)$ using Bray-Curtis similarity coefficient to construct the dendrogram. MVDISP was used to compare the stability of communities between the two stations. One-way ANOSIM was used to check whether the thermal plume would affect fish communities $(R<0.25$, barely separable at all; $R>0.5$, overlapping but clearly different; $R$
$>0.75$, well separated).

\section{RESULTS}

\section{Reef fishes}

A total number of 42 families and 134 species were recorded (Table 1). Among which, 39 families and 100 species were observed from experimental station and 33 families and 112 species were obtained from control station. Only 5 species were non-reef fish species i.e., $96.3 \%$ were reef species in which $91.8 \%$ were residential and $93.3 \%$ were demersal. Most fishes observed were in young or sub-adult stages. Diodon holocanthus, Abudefduf septemfasciatus, and A. vaigensis were the three species recorded every time and also the most abundant. Labridae is the most dominant family with the most numerous species. At control station, more wrasse species were recorded than that at experimental station but not for the rest species. Comparing the species composition between the two stations, 21 species only occurred at experimental station and 33 species at control station. Sorensen similarity coefficient was 0.738 which indicates that their species composition were very similar to each other. Although the species number was significantly higher at control station than at experimental station $(p<0.01$, ANOVA pair $\mathrm{T}$ test), no significant difference was found for total individual numbers (Table 2).

Clustering analysis showed that the community

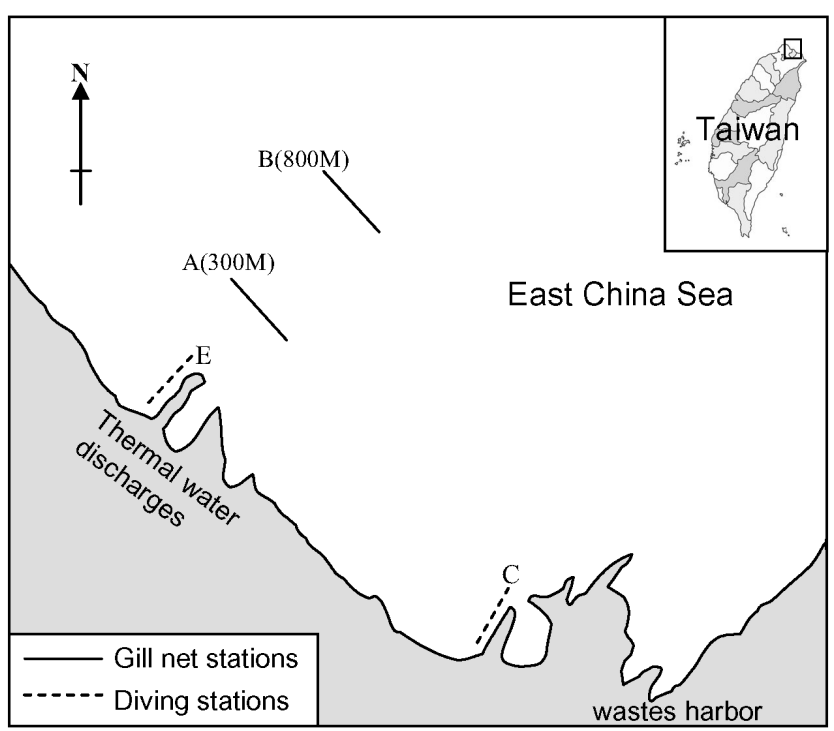

Fig. 1. The two sampling stations of underwater census by SCUBA divers, $\mathrm{C}$, control station, and $\mathrm{E}$, experimental staion along the jetty of outlet bay. The two three-layer drift net stations, A, experimental station about $300 \mathrm{~m}$ away from outlet area and $\mathrm{B}$, control station located about $800 \mathrm{~m}$ away. 
Table 1. Fish checklist and their individual numbers observed by diving at both experimental (E) and control (C) station from March 2001 to September 2004

\begin{tabular}{|c|c|c|c|c|c|c|c|c|c|}
\hline Family & Species & Guild & $\mathrm{E}$ & $\mathrm{C}$ & Family & Species & Guild & $\mathrm{E}$ & $\mathrm{C}$ \\
\hline \multirow[t]{5}{*}{ Acanthuridae } & Acanthurus dussumieri & $\mathrm{R}$ & 19 & 21 & & Thalassoma hardwickii & $\mathrm{R}$ & & 1 \\
\hline & Acanthurus nigrofuscus & $\mathrm{R}$ & & 1 & & Thalassoma lunare & $\mathrm{R}$ & 4 & 4 \\
\hline & Acanthurus xanthopterus & $\mathrm{R}$ & 29 & 32 & & Thalassoma lutescens & $\mathrm{R}$ & & 2 \\
\hline & Naso annularis & $\mathrm{R}$ & & 2 & & Xyrichtys dea & $\mathrm{R}$ & 1 & 2 \\
\hline & Prionurus scalprus & $\mathrm{R}$ & 4 & 33 & Leiognathidae & Leiognathus nuchalis & $\mathrm{V}$ & 7,350 & 50 \\
\hline \multirow[t]{10}{*}{ Apogonidae } & Apogon apogonides & $\mathrm{R}$ & 1 & & Lethrinidae & Lethrinus atkinsoni & $\mathrm{R}$ & 1 & 8 \\
\hline & Apogon aureus & $\mathrm{R}$ & 15 & & Lethrinidae & Lethrinus lentjan & $\mathrm{R}$ & & 2 \\
\hline & Apogon cookii & $\mathrm{R}$ & 109 & 315 & Lutjanidae & Lutjanus argentimaculatus & $\mathrm{R}$ & 2 & 1 \\
\hline & Apogon doederleini & $\mathrm{R}$ & 220 & 314 & & Lutjanus fulviflamma & $\mathrm{R}$ & 23 & 10 \\
\hline & Apogon fleurieu & $\mathrm{R}$ & 100 & & & Lutjanus fulvus & $\mathrm{R}$ & 1 & \\
\hline & Apogon nitidus & $\mathrm{R}$ & 217 & 68 & & Lutjanus gibbus & $\mathrm{R}$ & 1 & 21 \\
\hline & Apogon taeniophorus & $\mathrm{R}$ & 26 & 1 & & Lutjanus monostigma & $\mathrm{R}$ & 1 & \\
\hline & Archamia goni & $\mathrm{R}$ & & 275 & & Lutjanus quiquelineatus & $\mathrm{R}$ & & 1 \\
\hline & Archamia dispilus & $\mathrm{R}$ & 10 & 216 & & Lutjanus rivulatus & $\mathrm{R}$ & 9 & \\
\hline & Rhabdamia gracilis & $\mathrm{R}$ & 304 & 1,000 & & Lutjanus russellii & $\mathrm{R}$ & 3 & 10 \\
\hline Atherinidae & Hypoatherina woodwardi & $\mathrm{V}$ & 4,650 & 3,200 & & Lutjanus stellatus & $\mathrm{R}$ & 6 & 3 \\
\hline Belonidae & Tylosurus sp. & $\mathrm{V}$ & 2 & & & Lutjanus vitta & $\mathrm{R}$ & 2 & 12 \\
\hline \multirow{3}{*}{ Blenniidae } & Ecsenius lineatus & $\mathrm{R}$ & 6 & 1 & & Lutjanus kasmira & $\mathrm{R}$ & 3 & 2 \\
\hline & Ecsenius namiyei & $\mathrm{R}$ & & 1 & & Pterocaesio diagramma & $\mathrm{V}$ & 3 & 183 \\
\hline & Petroscirtes breviceps & $\mathrm{R}$ & 17 & 61 & Microdesmidae & Ptereleotris hanae & $\mathrm{R}$ & 13 & \\
\hline \multirow[t]{4}{*}{ Carangidae } & Alectis indicus & $\mathrm{V}$ & 1 & & Monacanthidae & Stephanolepis cirrhifer & $\mathrm{R}$ & & 4 \\
\hline & Caranx sexfasciatus & $\mathrm{V}$ & 8 & 1 & Mugilidae & Liza sp. & $\mathrm{V}$ & 65 & 11 \\
\hline & Seriola dumerili & $\mathrm{V}$ & 1 & & & Mugil cephalus & $\mathrm{V}$ & & 26 \\
\hline & Selaroides leptolepis & $\mathrm{V}$ & 278 & 340 & Mullidae & Parupeneus barberinus & $\mathrm{R}$ & & 4 \\
\hline \multirow[t]{8}{*}{ Chaetodotidae } & Coradion altivelis & $\mathrm{R}$ & & 1 & & Parupeneus ciliatus & $\mathrm{R}$ & 4 & 31 \\
\hline & Chaetodon auriga & $\mathrm{R}$ & 4 & 8 & & Parupeneus chrysopleuron & $\mathrm{R}$ & 4 & 12 \\
\hline & Chaetodon auripes & $\mathrm{R}$ & 14 & 33 & & Parupeneus indicus & $\mathrm{R}$ & 51 & 74 \\
\hline & Chaetodon lunula & $\mathrm{R}$ & & 2 & & Parupeneus multifasciatus & $\mathrm{R}$ & & 2 \\
\hline & Chaetodon melannotus & $\mathrm{R}$ & & 1 & & Parupeneus pleurostigma & $\mathrm{R}$ & & 1 \\
\hline & Chaetodon octofasciatus & $\mathrm{R}$ & 2 & & & Upeneus tragula & $\mathrm{R}$ & 27 & 27 \\
\hline & Chaetodon vagabundus & $\mathrm{R}$ & & 2 & & Upeneus japonicus & $\mathrm{R}$ & 17 & 2 \\
\hline & Heniochus acuminatus & $\mathrm{R}$ & 8 & 27 & Nemipteridae & Scolopsis monogramma & $\mathrm{R}$ & 3 & 1 \\
\hline Cheilodactylidae & Cheilodactylus zonatus & $\mathrm{R}$ & 5 & 2 & & Scolopsis vosmeri & $\mathrm{R}$ & 29 & 11 \\
\hline Cichlidae & Orechromis hybrid & $\mathrm{R}$ & & 3 & Oplegnathidae & Oplegnathus fasciatus & $\mathrm{R}$ & 1 & 11 \\
\hline Diodontidae & Diodon holcanthus & $\mathrm{R}$ & 84 & 102 & Ostraciidae & Ostracion cubicus & $\mathrm{R}$ & 2 & 3 \\
\hline Fistulariidae & Fistularia commersonii & $\mathrm{R}$ & 11 & 29 & Pempheridae & Pempheris oualensis & $\mathrm{R}$ & 5 & 472 \\
\hline Gerreidae & Gerres oyena & $\mathrm{R}$ & 3 & 21 & Pinguipedidae & Parapercis xanthozona & $\mathrm{R}$ & 7 & \\
\hline \multirow[t]{2}{*}{ Gobiidae } & Valenciennea muralis & $\mathrm{R}$ & 16 & & Plotosidae & Plotosus lineatus & $\mathrm{R}$ & 1 & 60 \\
\hline & Istigobius campbelli & $\mathrm{R}$ & 16 & 10 & Pomacanthidae & Chaetodontoplus septentrionalis & $\mathrm{R}$ & 1 & 3 \\
\hline \multirow[t]{6}{*}{ Haemulidae } & Parapristipoma trilineatum & $\mathrm{R}$ & 2,026 & 52 & Pomacanthidae & Pomacanthus semicirculatus & $\mathrm{R}$ & & 6 \\
\hline & Plectorhinchus cinctus & $\mathrm{R}$ & 3 & & Pomacentridae & Abudefduf septemfasciatus & $\mathrm{R}$ & 214 & 186 \\
\hline & Plectorhinchus flavomaculatus & $\mathrm{R}$ & 2 & 1 & & Abudefduf sexfasciatus & $\mathrm{R}$ & 16 & 119 \\
\hline & Plectorhinchus nigrus & $\mathrm{R}$ & 3 & 1 & & Abudefduf sordidus & $\mathrm{R}$ & 49 & 28 \\
\hline & Plectoorhinchus pictum & $\mathrm{R}$ & 2 & 4 & & Abudefduf vaigiensis & $\mathrm{R}$ & 1,112 & 1,054 \\
\hline & Pomadasys quadrilineatus & $\mathrm{R}$ & 3,281 & 2,570 & & Amphiprion clarkii & $\mathrm{R}$ & 4 & \\
\hline \multirow[t]{4}{*}{ Kyphosidae } & Girella mezina & $\mathrm{R}$ & & 5 & & Chromis fumeus & $\mathrm{R}$ & 22 & 39 \\
\hline & Girella punctata & $\mathrm{R}$ & 1 & 10 & & Chromis notatus & $\mathrm{R}$ & & 52 \\
\hline & Kyphosus cinerascens & $\mathrm{R}$ & & 10 & & Neopomacentrus cyanomus & $\mathrm{R}$ & 36 & 101 \\
\hline & Microcanthus strigathus & $\mathrm{R}$ & 208 & 74 & & Pomacentrus coelestis & $\mathrm{R}$ & 130 & 152 \\
\hline \multirow[t]{7}{*}{ Labridae } & Bodianus diana & $\mathrm{R}$ & & 1 & & Stegastes altus & $\mathrm{R}$ & 3 & 21 \\
\hline & Anampses caeruleopunctatus & $\mathrm{R}$ & & 4 & Scaridae & Chlorurus microrhinos & $\mathrm{R}$ & & 2 \\
\hline & Cheilio inermis & $\mathrm{R}$ & & 1 & & Scarus rubroviolaceus & $\mathrm{R}$ & 3 & 3 \\
\hline & Choserodon azurio & $\mathrm{R}$ & 1 & 2 & Scatophagidae & Scatophagus argus & $\mathrm{R}$ & 1 & \\
\hline & Coris dorsomacula & $\mathrm{R}$ & 42 & 67 & Scorpaenidae & Pterois volitans & $\mathrm{R}$ & 1 & \\
\hline & Coris gaimard & $\mathrm{R}$ & 3 & 3 & Serranidae & Cephalopholis boenak & $\mathrm{R}$ & 6 & \\
\hline & Halichoeres argusr & $\mathrm{R}$ & 1 & 5 & & Diploprion bifasciatum & $\mathrm{R}$ & & 6 \\
\hline Labridae & Halichoeres marginatus & $\mathrm{R}$ & & 6 & & Epinephelus quoyanus & $\mathrm{R}$ & 1 & 3 \\
\hline & Halichoeres melanochir & $\mathrm{R}$ & 27 & 18 & & Grammistes sexlineatus & $\mathrm{R}$ & & 1 \\
\hline & Halichoeres nebulosus & $\mathrm{R}$ & & 6 & Siganidae & Siganus fuscescens & $\mathrm{R}$ & 13,204 & 13,103 \\
\hline & Halichoeres nigrescens & $\mathrm{R}$ & 35 & 58 & & Siganus guttatus & $\mathrm{R}$ & & 6 \\
\hline & Halichoeres orientalis & $\mathrm{R}$ & & 9 & Sparidae & Acanthopagrus schlegeli & $\mathrm{R}$ & 2 & \\
\hline & Halichoeres poecilopterus & $\mathrm{R}$ & 25 & 35 & Sphyraenidae & Sphyraena flavicauda & V & 334 & 527 \\
\hline & Labroides dimidiatus & $\mathrm{R}$ & 11 & 20 & Synodontidae & Trachinocephalus myops & $\mathrm{R}$ & 1 & \\
\hline & Pseudocoris ocellata & $\mathrm{R}$ & 44 & 30 & Tetraodontidae & Arothron sp. & $\mathrm{R}$ & 1 & \\
\hline & Pseudolabrus eoethinus & $\mathrm{R}$ & 12 & 74 & Zancidae & Zanclus cornutus & $\mathrm{R}$ & & 3 \\
\hline & Pteragogus enneacantus & $\mathrm{R}$ & 1 & & Zanclidae & Zanclus cornutus & $\mathrm{R}$ & & 3 \\
\hline & Stethojulis terina & $\mathrm{R}$ & 74 & 188 & & & & & \\
\hline & Suezichthys gracilis & $\mathrm{R}$ & 8 & 5 & & Total species number & & 100 & 112 \\
\hline & Thalassoma amblycephalum & $\mathrm{R}$ & & 1 & & Total individual number & & 34,770 & 25,852 \\
\hline
\end{tabular}

In the column of habitat guild, $\mathrm{R}$ means residential and $\mathrm{M}$, migratory or transient. 
structure between the two stations could not be distinguished (Fig. 2). The separate group of three samples of E0409, E0304 and C0304 was due to very low water visibility $(<3 \mathrm{~m})$ while diving which let the observation data invalid. If we took off these three samples, oneway ANOSIM analysis still could not show significant difference $(R=0.282, p=0.001)$. Yearly difference among fish assemblages did not exist either (one-way ANOSIM test, E station $R=0.192(p=0.035), C$ station $R=0.186(\mathrm{P}=0.04))$. Nevertheless, dispersion index at control station was slightly lower than that at experimental station (MVDISP test $\mathrm{E}=1.052$ vs. $\mathrm{C}=0.956$ ). If we deleted those pelagic or transient species, there was still no significant difference detected between the two stations $(R=0.319, p=0.001)$. No significant difference of community structure was found among different years (one-way ANOSIM test $\mathrm{E}$ station $\mathrm{R}=$ $0.192(\mathrm{P}=0.035), \mathrm{C}$ station $R=0.186(\mathrm{P}=0.04)$. Dispersion index was still slightly lower at the control station than at the experimental station (MVDISP test E $=1.10$ vs. $\mathrm{C}=0.911$ ). In other words, the stability of reef fish assemblage was lower in the thermal waters than in the ambient waters.

\section{Demersal and pelagic fishes}

Table 3 listed all 33 families and 64 species in total from 16 sampling times at the two stations. From the $300 \mathrm{~m}$ experimental station, 26 families and 43 species were recorded, and 24 families and 37 species from the control station. Among 64 species, 21 were non-reef species $(32.83 \%), 18.8 \%$ were pelagic, the rest of $43.87 \%$ were still reef fish species. Nevertheless, the total number of individuals or biomass of fish samples belong to pelagic or migratory species, such as Carangidae was the most diverse family with 8 species,

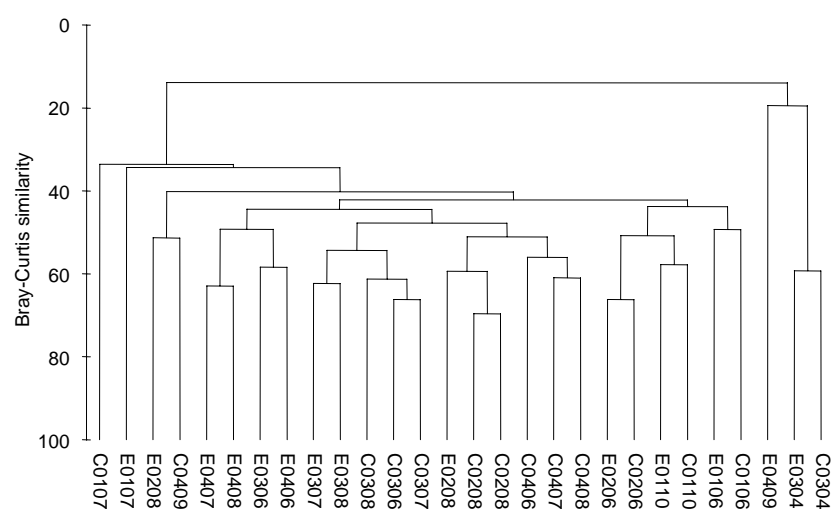

Fig. 2. UPGMA dendrogram for monitoring reef fish assemblages at both experimental $(E)$ and control $(C)$ station near the outlet area of the $2^{\text {nd }}$ NPP during March 2001 to September 2004 (The first two digits are abbrev. of $\mathrm{AD}$, the latter two digits are months). and Arius maculates was the highest caught in both total individual number and biomass.

Comparing the species composition, there were 23 species which only appearing at $300 \mathrm{~m}$ station and 16 species only appeared at $800 \mathrm{~m}$ station. The Sorensen similarity coefficient value was 0.5 , even lower than the values between the two reef fish stations. Table 4 lists the total species number, individual number and body weight between the two drift-net stations which showed no significant difference.

The clustering dendrogram of Fig. 3 only showed the fish assemblage in autumn, i.e., October and November, was different from the fish assemblages in other seasons. This was due to high catches of Platyrhina sinensis, Alectis indicus, Diodon holocanthus and Alectis ciliaris etc, in these two months. Even we delete the data of these two months, no significant difference was found between the two stations (Global test $R=0, p=$ 0.432). This result indicates that thermal plume did not affect the community structure of either pelagic or demersal fishes.

\section{DISCUSSION}

For reef fishes, although our sampling stations of diving were moved back to the areas which are very close to the outlet bay, even along the jetty of outlet bay for monitoring reef fishes, no significant difference of fish assemblages has been found between effluent area and non-effluent area. This is because the warmer waters only existed in the surface layer, less than $1.5 \mathrm{~m}$. Most of fishes observed at experimental station were reef fishes associated with reef bottom, around 5-6 meters depth, which should not be effected directly by the thermal plume. Figure 4 show that there was no temperature difference in the waters below $1.5 \mathrm{~m}$ but only existed in the surface layer. Indeed this warmer surface waters can attract some thermophilic species especially for those inshore migratory post-larval or juveniles, such as Terapon jurba and Liza macrolepis, the two famous malformed species caused by high water temperature at the $2^{\text {nd }}$ NPP, which gathering or even living inside the outlet bay [7,9]. However, these two

Table 2. The result of paired $T$ test on the total number of species and individual numbers of ref fishes between experimental and control

\begin{tabular}{lrll}
\hline & $T$ value & $P$ value & $d f$ \\
\hline Species No. & -3.0783 & $0.0088^{* *}$ & 13 \\
Individual No. & 1.2544 & 0.2318 & 13 \\
\hline
\end{tabular}

$* p<0.05$.

$* * p<0.01$ 
Table 3. Checklist and their individual numbers caught by drift net at both experimental (A) and control (B) station during March 2001 to July 2004

\begin{tabular}{|c|c|c|c|c|c|c|c|c|c|c|c|c|c|}
\hline & & & & Total & A & B & & & & & Total & A & B \\
\hline Family & Species & Stage & Habitat & Wight (g) & NO. & NO. & Family & Species & Stage & Habitat & Wight (g) & NO. & NO. \\
\hline \multirow[t]{2}{*}{ Acanthuridae } & Acanthurus dussumieri & A & $\mathrm{R}$ & 445 & 1 & 1 & Lutjanidae & Lutjanus fuliflamma & A & $\mathrm{R}$ & 376 & 2 & \\
\hline & Prionurus scalprus & A & $\mathrm{R}$ & $4,148.6$ & & 5 & Monacanthidae & Aluterus monoceros & A & $\mathrm{R}$ & $1,501.6$ & & 2 \\
\hline Ariidae & Arius maculatus & A & S & $26,637.7$ & 32 & 23 & Monacanthidae & Chaetodermis penicilligerus & A & $\mathrm{R}$ & 130 & 1 & \\
\hline \multirow[t]{8}{*}{ Carangidae } & Alectis ciliaris & $\mathrm{J}$ & $\mathrm{R}$ & $1,492.1$ & 2 & 3 & Narcinidae & Narcine timlei & A & $S$ & 1,172 & 1 & \\
\hline & Alectis indicus & $\mathrm{J}$ & $\mathrm{R}$ & $11,192.2$ & 9 & 6 & Oplegnathidae & Oplegnathus fasciatus & A & $\mathrm{R}$ & $2,299.7$ & 1 & 4 \\
\hline & Caranx papuensis & A & $\mathrm{R}$ & $2,090.6$ & 6 & 4 & & Oplegnathus punctatus & A & $\mathrm{R}$ & 347.3 & 1 & \\
\hline & Carangoides dinema & A & $S$ & 316.8 & 1 & & Osmeridae & Ostracion cubicus & A & $\mathrm{R}$ & $4,985.2$ & 7 & 11 \\
\hline & Carangoides hedlandensis & A, J & S & 50.9 & 1 & & Paralichthyidae & Paralichthys olivaceus & A & $\mathrm{S}$ & 267 & 1 & \\
\hline & Decapterus muroadsi & A & S & 96.3 & 1 & 1 & Pempheridae & Pempheris vanicolensis & A & $\mathrm{R}$ & 215 & 1 & \\
\hline & Megalaspis cordyla & A & $S$ & $1,044.3$ & & 1 & Pomacanthidae & Pomacanthus semicirculatus & A, J & $\mathrm{R}$ & 5,130 & 1 & \\
\hline & Seriola dumerili & $\mathrm{J}$ & $\mathrm{R}$ & 1825 & 2 & & Rachycentridae & Rachycentron canadum & $\mathrm{J}$ & $\mathrm{S}$ & $1,708.9$ & & 1 \\
\hline \multirow[t]{2}{*}{ Cheilodactylidae } & Cheilodactylus zonatus & A & $\mathrm{R}$ & $1,397.6$ & & 4 & Rhinobatidae & Platyrhina sinensis & A & $\mathrm{S}$ & $2,795.9$ & 12 & 9 \\
\hline & Cheilodactylus quadricornis & A & $\mathrm{R}$ & 235.6 & & 1 & & Rhinobatos schlegeli & A & $\mathrm{S}$ & 2,445 & & \\
\hline \multirow[t]{4}{*}{ Chaetodontidae } & Chaetodon auripes & A & $\mathrm{R}$ & 370 & & 2 & Scaridae & Scarus ghobban & A & $\mathrm{R}$ & 576 & & 1 \\
\hline & Coradion altivelis & A & $\mathrm{R}$ & 566 & 4 & 2 & Sciaenidae & Arygrosomus japonicus & A & $\mathrm{S}$ & $5,121.2$ & 6 & 4 \\
\hline & Chaetodon modestus & A & $\mathrm{R}$ & 50.3 & 1 & & Sciaenidae & Johnius distinctus & A & $\mathrm{S}$ & 124.4 & 1 & \\
\hline & Chaetodon wiebeli & A & $\mathrm{R}$ & 110 & 1 & & & Pennahia macrocophalus & A & $\mathrm{S}$ & 617.3 & 4 & 2 \\
\hline Clupeidae & Sardinella sindensis & A & $S$ & 15.2 & & 1 & Scombridae & Scomber japonicus & A & $\mathrm{S}$ & 320 & 4 & \\
\hline \multirow[t]{2}{*}{ Dasyatidae } & Dasyatis bennetti & A & $S$ & 898.6 & 2 & & Scorpaenidae & Pterois volitans & A & $\mathrm{R}$ & 500 & & 1 \\
\hline & Dasyatis kuhlii & A & $\mathrm{R}$ & 680 & 1 & & & Scorpaenopsis cirrhosa & A & $\mathrm{R}$ & 118 & 1 & \\
\hline Diodontidae & Diodon holocanthus & A & $\mathrm{R}$ & 4,256 & 5 & 12 & & Scorpaenopsis diabolus & A & $\mathrm{R}$ & 75.2 & & 1 \\
\hline Elopidae & Elops haw aiensis & A & $S$ & $2,048.2$ & & 3 & & Sebastiscus marmoratus & A & $\mathrm{R}$ & 365.6 & 1 & 2 \\
\hline \multirow[t]{4}{*}{ Haemulidae } & Diagramma pictum & A & $\mathrm{R}$ & $8,220.3$ & 9 & 9 & Serranidae & Cephalopholis boenak & A & $\mathrm{R}$ & 335.6 & 1 & 2 \\
\hline & Hapalogenys mucronatus & A & S & $2,615.7$ & 6 & 13 & & Epinephelus chlorostigma & A & $\mathrm{R}$ & 927.9 & & 3 \\
\hline & Parapristipoma trilineatum & $\mathrm{A}, \mathrm{J}$ & $\mathrm{R}$ & 2,074 & 9 & 4 & Siganidae & Siganus fuscescens & $\mathrm{A}, \mathrm{J}$ & $\mathrm{R}$ & 512.3 & 2 & 1 \\
\hline & Plectorhinchus cinctus & A & $\mathrm{R}$ & 1,500 & 2 & & Sparidae & Acanthopagrus schlegelii & A & $\mathrm{S}$ & 213.3 & 1 & \\
\hline \multirow[t]{2}{*}{ Holocentridae } & Sargocentron melanospilos & A & $\mathrm{R}$ & $1,112.3$ & 3 & 1 & & Rhabdosargus sarba & A & $\mathrm{S}$ & 931.9 & 1 & 1 \\
\hline & Sargocentron rubrum & A & $\mathrm{R}$ & 398 & & 2 & Triakidae & Mustelus griseus & $\mathrm{J}$ & $\mathrm{S}$ & 1,700 & & 1 \\
\hline \multirow[t]{2}{*}{ Kyphosidae } & Girella punctata & A & $\mathrm{R}$ & 305 & 1 & & Uranoscopidae & Ichthyscopus lebeck & $\mathrm{J}$ & $\mathrm{S}$ & 125 & 1 & \\
\hline & Microcanthus strigatus & A & $\mathrm{R}$ & 275.8 & & 2 & & Total species number & & & & 43 & 37 \\
\hline Labridae & Choerodon azurio & A & $\mathrm{R}$ & 53.3 & & 1 & & Total individual number & & & & 149 & 143 \\
\hline Lethrinidae & Lethrinus nebulosus & A & $\mathrm{R}$ & 494.5 & 1 & & & Total weight & & & 113428 & & \\
\hline
\end{tabular}

In the column of Morphological stage: A means adult, and J, juveniles; in Habitat Column; R, rocky and S, sandy.

Table 4. The result of paired $T$ test on the total number of species, individual numbers and biomass of pelagic or demersal fishes caught between experimental and control station

\begin{tabular}{lccc}
\hline & $T$ value & $P$ value & $d f$ \\
\hline Total species number & 1.0194 & 0.3232 & 16 \\
Total individual number & -0.3735 & 0.7137 & 16 \\
Total weight & -1.7087 & 0.1068 & 16
\end{tabular}

$* p<0.05$.

$* * p<0.01$.

species could not be easily observed and recorded by divers because most of them lived inside the outlet bay with sandy bottom.

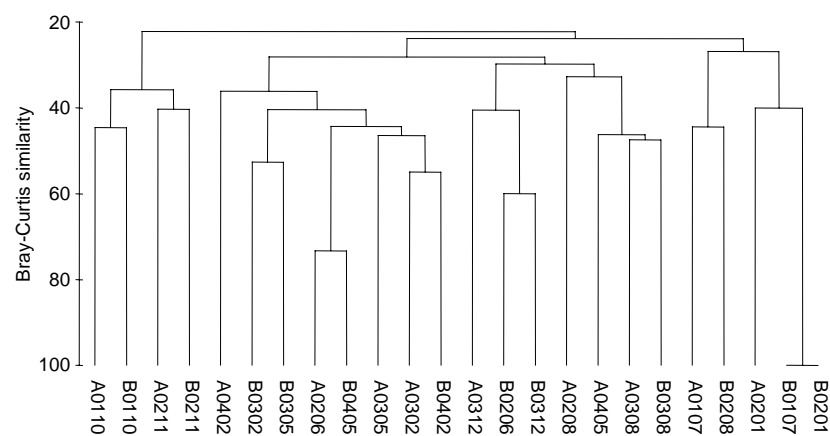

Fig. 3. UPGMA dendrogram for monitoring pelagic or demersal fish assemblages at experimental (A) and control (B) station $300 \mathrm{~m}$ and $800 \mathrm{~m}$ away respectively from the outlet area of the $2^{\text {nd }} \mathrm{NPP}$ from July 2001 to May 2004 (The first two digits are abbrev. of AD, the latter two digits are months). 
(a) Surface

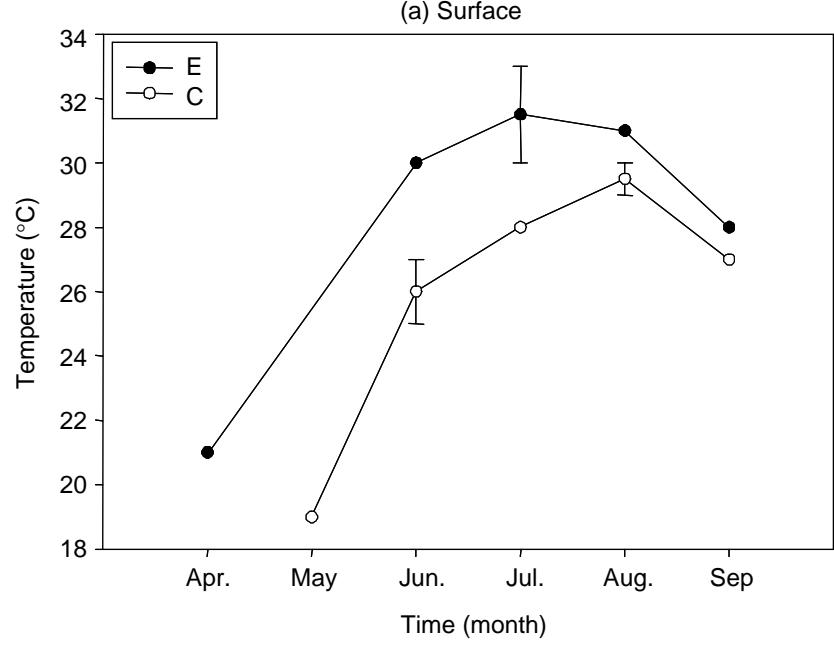

(b) Underwater $1.5 \mathrm{M}$

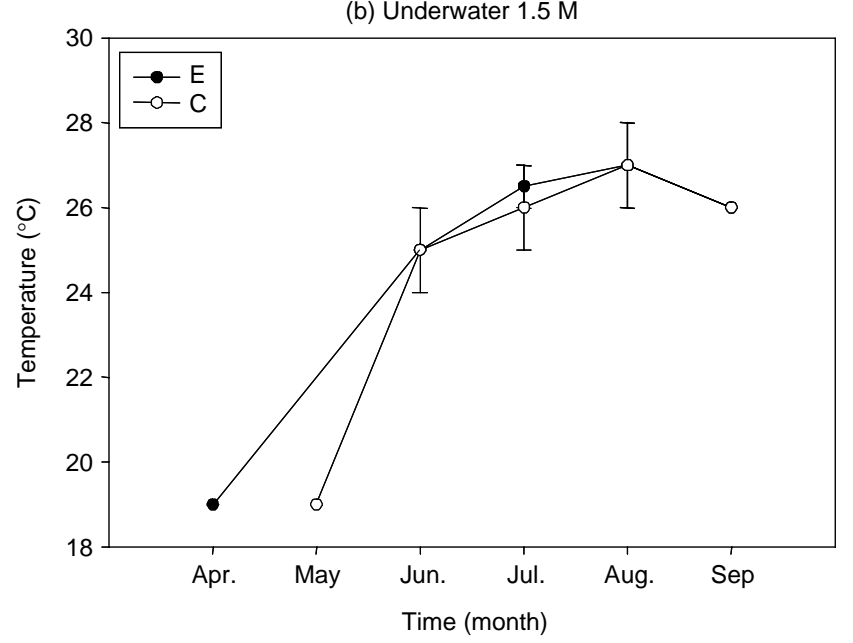

Fig. 4. Water temperature differences between the experimental and the control stations in the upper surface layer or $1.5 \mathrm{~m}$ below the surface.

Are there any species which could be possible related to the temperature difference? Table 5 lists some fish species which were different between the two stations. That the two herbivorous species of Petroscirtes breviceps and Stegastes altus were more abundant at the control station than at the experimental station might be because seaweed growth was better in cooler water than in warmer water [8]. As to zooplankton feeder of Parapristipoma trilineatum and omnivores Microcanthus strigatus, that they were more abundant in warmer waters which might be due to more food particles or less active zooplankton stirred up by thermal plume at experimental station. But three species of carnivores, Apogon cookii, Stethojulis terina and Parupeneus ciliatus, and three species of omnivores, Prionurus scalprus, Pempheris oualensis and Abudefduf
Table 5. The list some fish species which were quantitatively different between experimental (E) and control (C) stations $(Z$, zooplankton feeder; $O$, omnivores; $C$, carnivores; $\mathbf{H}$, herbivores)

\begin{tabular}{lcrr}
\hline Species & Food guild & \multicolumn{1}{c}{ E } & \multicolumn{1}{c}{ C } \\
\hline Parapristipoma trilineatum & $\mathrm{Z}$ & 2026 & 52 \\
Microcanthus strigatus & $\mathrm{O}$ & 208 & 74 \\
Apogon cookii & $\mathrm{C}$ & 109 & 315 \\
Stethojulis terina & $\mathrm{C}$ & 74 & 188 \\
Neopomacentrus cyanomus & $\mathrm{Z}$ & 36 & 101 \\
Petroscirtes breviceps & $\mathrm{H}$ & 17 & 61 \\
Abudefduf sexfasciatus & $\mathrm{O}$ & 16 & 119 \\
Archamia dispilus & $\mathrm{Z}$ & 10 & 216 \\
Heniochus acumnatus & $\mathrm{Z}$ & 8 & 27 \\
Pempheris oualensis & $\mathrm{C}$ & 5 & 472 \\
Prionurus scalprus & $\mathrm{O}$ & 4 & 33 \\
Parupeneus ciliatus & $\mathrm{C}$ & 4 & 31 \\
Stegastes altus & $\mathrm{H}$ & 3 & 21 \\
\hline
\end{tabular}

sexfasciatus were more abundant at the control station than at the experimental station. It was probably due to microhabitat difference rather than water difference because the above fishes are mostly living near bottom where the water temperature are similar to each others for these two stations.

For non-reef associate pelagic or demersal fishes outside the thermal effluent, no significant spatial difference between $300 \mathrm{~m}$ and $800 \mathrm{~m}$ away from outlet bay was obtained except the seasonal difference in the winter. This result probably because of that these two drift net stations were not so far away from each other and the temperature gradient (approximately less than $3^{\circ} \mathrm{C}$ between the two stations) was not so large enough to attract fishes and form distribution pattern, especially for the swimming ability and migratory range of nonreef and non-residential fishes that swim at the surface layer and have larger migratory range.

However, every weekend in the summer season, there are so many anglers crowded in the jetty of the outlet bay to fish those thermophilic milkfish or needlefishes. This thermal attraction phenomenon should be due to the fact that the temperature gradient here is much greater than the diffusion area outside the bay. Also, strong turbulent which probably mixed with more food particles in the plume within $100 \mathrm{~m}$ range probably can create stronger attraction effect than outside $100 \mathrm{~m}$ apart from the mouth of outlet bay.

In conclusion, based on our three and half years monitoring data on reef and non-reef fish assemblages in the waters surrounding the outlet area of the $2^{\text {nd }}$ NPP, the thermal plume or higher water temperature in the surface layer did not change the fish assemblages 
significantly. This conclusion is the same as the results of Calanoid [16].

\section{REFERENCES}

1. Cironi R., Ioannili, E., and Vitali, R., Assessment of Effects of Coastal Power Plants on Marine Biological Resources in Italy, E \& Fn Spon, London, pp. 313-329 (1995).

2. Clarke, K.R. and Gorley, R.N., PRIMER v5: User Manual/ Tutorial, PRIMER-E, Plymouth Marine Laboratory, UK, pp. 1-91 (2001).

3. Huang, C.C., Hung, T.C., and Fan, K.L., "Nonbiological Factors Associated with Coral Bleaching in Shallow Water Near the Outlet of the Third Nuclear Power Plant, Southern Taiwan," UNEP Regional Seas Reports Stud., Vol. 147, pp. 205-224 (1992).

4. Hung, T.C., Huang, C.C., and Shao, K.T., "Ecological Survey of Coal Water Adjacent to Nuclear Power Plants in Taiwan," Chem. Ecol., Vol. 15, pp. 129-142 (1998).

5. Hwang, D.F., Chien, L.T., Shao, K.T., and Jeng, S.S., "Levels of Heavy Metals and Vitamin C in Deformed Thornfish Found in Thermal Waters and Effect of Vitamin C on Deformation of Thornfish," Fish. Sci., Vol. 64, No. 2, pp. 291-294 (1998).

6. Su, J.C., Hung, T.C., Chiang, Y.M., Tan, T.H., Chang, K.H., Lee, C.T., and Chang, H.T., "The Final Report for the Assessment of Ecological Impact on the Operation of the Nuclear Power Plant along the Northern Coast of Taiwan (July 1974- June 1984)," SCOPE/Academia Sinica, Vol. 29, pp. 1-151 (1984).

7. Jan, R.Q., Chen, J.P., Lin, C.Y., and Shao, K.T., "Longtern Monitoring of the Coral Reef Fishes Communities Around a Nuclear Power Plant," Aquat. Ecol., Vol. 35, No. 2, pp. 233-243 (2001).

8. Kakinuma, M., Shibahara, N., Ikeda, H., Maegawa, M., and Amano, H., "Thermal Stress Responses of a Sterile Mutant of Ulva Pertusa (Chlorphyta)," Fish. Sci., Vol. 67, pp. 287-294 (2001).
9. Lin, Y.H., "The Relation Between Malformed Fishes and the Temperature of Thermal Discharges at the $2^{\text {nd }}$. Nuclear Power Plant on the Northern Coast of Taiwan," M.S. Thesis, Institute of Marine Biology, National Taiwan Ocean University, Keelung, Taiwan (1995).

10. Sandstrom, O., Neuman, E., and Thoresson, G., "Effects of Temperature on Life History Variables in Perch," $J$. Fish Biol., Vol. 47, pp. 652-670 (1995).

11. Su, J.C., Hung, T.C., Chiang, Y.M., Tan, T.H., Chang, K. H., Shao, K.T., Huang, P.P., Lee, K.T., Huang, C.C., Huang, C.Y., Fan, K.L., and Yeh, S.Y., "An Ecological Survey on the Waters Adjacent to the Nuclear Power Plant in Southern Taiwan: The Preliminary Report for the Assessment of Ecological Impact of the Operation of the Power Plant (July 1979- June 1988)," SCOPE/ Academia Sinica, Vol. 59, pp. 1-394 (1988).

12. Suresh, K., Ahamed, M.S., Durairaj, G., and Nair, K.V. K., "Impact of Power Plant Heated Effluent on the Abundance of Sedentary Organism, off Kalpakkam, East Coast of India," Hydrobiologia, Vol. 268, pp. 109114 (1993).

13. Suresh K., Ahamed, M.S., and Durairaj, G., "Environmental Physiology of the Mole Crab Emerita asiatica, at a Power Plant Discharge Area on the East Coast of India," Environ. Poll., Vol. 88, pp.133-136 (1995).

14. Symthe, A.G. and Sawyko, P.M., "Field and Laboratory Evaluation of the Effects of "Cold Chock" on Fish Resident in and Around a Thermal Discharge: An Overview," Environ. Sci. Pol., Vol. 3, pp.225 -232 (2000).

15. Verlaque, M., Giraud, G., and Boudouresque, C.F., "Effects of a Thermal Power Plant on the Mediterranean Marine Phythobenthos: The Area of High Frequency Temperature Charges," Bot. Mar., Vol. 24, pp.69-87 (1981).

16. Wong, C.K., Hwang, J.S., and Chen, Q.C., "Taxonomic Composition and Grazing Impact of Calanoid Copepods in Coastal Waters near Nuclear Power Plants in Northern Taiwan,” Zool. Stud., Vol. 37, No. 4, pp. 330-339 (1998). 